\title{
In vivo tunable CRISPR mediates efficient somatic mutagenesis to generate tumor models
}

\begin{abstract}
Dear Editor,
CRISPR/Cas9 has revolutionized genome editing technology due to its simplicity and robustness (Mali et al., 2013). Several inducible CRISPR/Cas9 systems recently developed make spatiotemporal genome editing possible (Konermann et al., 2013; Balboa et al., 2015; Dow et al., 2015; Zetsche et al., 2015; Liu et al., 2016; Kleinjan et al., 2017; Maji et al., 2017; Senturk et al., 2017; Lu et al., 2018). However, whether these inducible CRISPR/Cas9 systems can mediate efficient in vivo somatic mutagenesis for tumor modeling remains to be tested. Destabilizing domain (DD), a member of the post-translationally inducible elements, can be fused to Cas9 protein to construct an inducible CRISPR/ Cas9 system (Senturk et al., 2017). The DD tag guides newly synthesized DD-Cas9 protein to the proteasome for degradation, which can be abrogated by ligand trimethoprim (TMP) (Fig. 1A) (Iwamoto et al., 2010; Sando et al., 2013). Because of the convenience of TMP delivery and no endogenous targets found for TMP in mammals, TMP-inducible Cas9 stabilization would be extremely attractive for achieving post-translationally tunable genome editing in vivo.
\end{abstract}

To obtain an efficient and tightly controlled DD-Cas9, we created five variants with distinct configurations, all on a piggyBac transposon plasmid backbone (Fig. 1B). We next determined the activities of these Cas 9 variants by targeting three human genes (TET1, TET2 and TET3) by T7 Endonuclease I (T7EI) assay (Figs. 1C, 1D and S1). In the presence or absence of TMP, no insertion/deletion (indel) was detected in the non-transfected (NT) control group (Fig. 1D). Among these five variants, F5D (DD fused to $\mathrm{N}$-terminal of Cas9 protein) displayed significantly higher activity than other variants when TMP was added, but demonstrated no activity in the absence of TMP (Fig. 1D). Hence, the F5D construct was used in all subsequent experiments. To optimize the working concentration of TMP, we examined Cas9 protein expression from F5D under eight TMP concentrations. At $0.01 \mu \mathrm{mol} / \mathrm{L}$ TMP, stabilized Cas9 protein started to show up. As TMP concentration increased, Cas 9 protein level increased gradually as well and reached a plateau at $1 \mu \mathrm{mol} / \mathrm{L}$ TMP (Fig. 1E). Therefore, $1 \mu \mathrm{mol} / \mathrm{L}$ TMP was used in all subsequent in vitro studies. Finally, we assayed the kinetics of DD mediated protein degradation. Cells were treated with TMP for 1, 2, 6, 12, 24, 48, $72 \mathrm{~h}$ and harvested for Cas 9 detection by Western blot. As soon as 2 $\mathrm{h}$ after TMP treatment, rapid Cas9 stabilization was detected and became undetectable $12 \mathrm{~h}$ after TMP withdrawal (Fig. 1F). In summary, these results demonstrated that F5D is highly efficient for in vitro genome editing and the DDCas 9 is tightly controlled by TMP at the protein level.

Our previous work demonstrated that tail vein injection of sgRNAs for Cdkn2a and Trp53, an hNRAS ${ }^{\mathrm{G} 12 \mathrm{~V}}$ overexpression plasmid, along with Cas9 expression plasmid achieved almost $100 \%$ liver tumorigenesis in mice (Xu et al., 2017). To determine whether DD-Cas9 works in vivo in a tunable manner, 26 mice were randomly separated into four groups and injected through tail vein with different plasmid combination (Table 1 and Fig. S2) to induce tumors (Fig. 2A). When examined six weeks after injection, mice in group $A$ (no F5D, no TMP), group B (no F5D, +TMP) and group C (F5D, no TMP) were all tumor-free (Fig. 2B). In group D (F5D, +TMP), liver tumors of various sizes with strong GFP fluorescence were found in eight of ten injected mice (Fig. 2B). These data suggested that F5D could induce tumors in a tunable manner as anticipated. Next, we performed T7EI assay and confirmed the successful gene targeting of both Cdkn2a and Trp53 in tumor tissues (Figs. 2C and S2B). In addition, TA-cloning and Sanger sequencing confirmed indel mutations at the two genomic loci (Fig. 2D). Finally, we carried out histological analysis and proliferation detection via H\&E and Ki67 staining respectively. Compared with normal liver sections, tumors induced showed a tubular growth morphology and were highly proliferative (Fig. 2E). Taken together, these results indicated that DD-Cas9 can inducibly mediate efficient liver tumorigenesis in adult mice, and DD-Cas9 can be exploited in vivo to engineer endogenous genes in an inducible manner.

TMP is inexpensive, non-toxic and can penetrate the placental barrier and the blood-brain barrier. All these advantages make TMP ideal to stabilize DD tagged proteins in vivo (Iwamoto et al., 2010). In the current study, we fused 


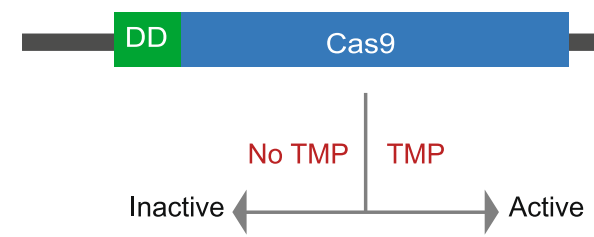

C

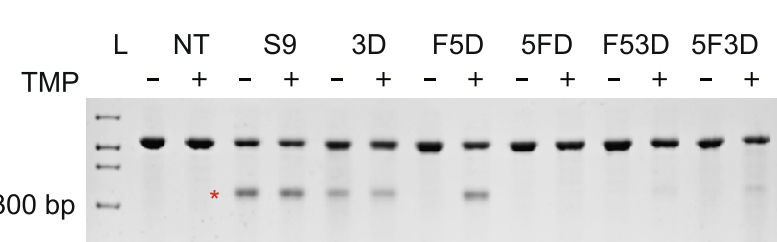

B

PB3

S9

$3 \mathrm{D}$

F5D

$5 F D$

F53D

$5 F 3 D$
CBh Flag NLS

CBh Flag NLS

CBh-Flag DD NLS

CBh DD Flag NLS

CBh Flag DD NLS

CBh DD Flag NLS

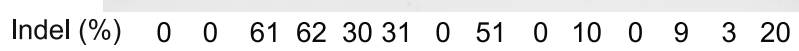

D

TET1

TET2

TET3
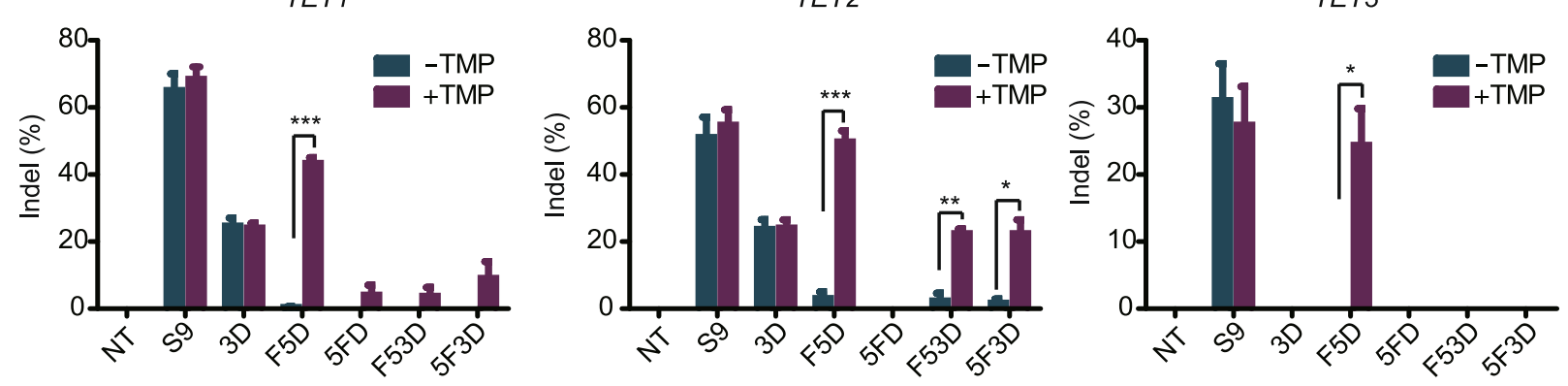

E

F

$\operatorname{TMP}(\mu \mathrm{mol} / \mathrm{L}) \quad \mathrm{NT} \quad 0 \quad 0.01 \quad 0.1 \quad 0.5 \quad 1 \quad 10 \quad 50 \quad 100500$

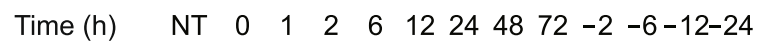

Anti-Flag

$-\infty 00000$

Anti-Flag

Anti- $\beta$-Tubulin

Anti- $\beta$-Tubulin
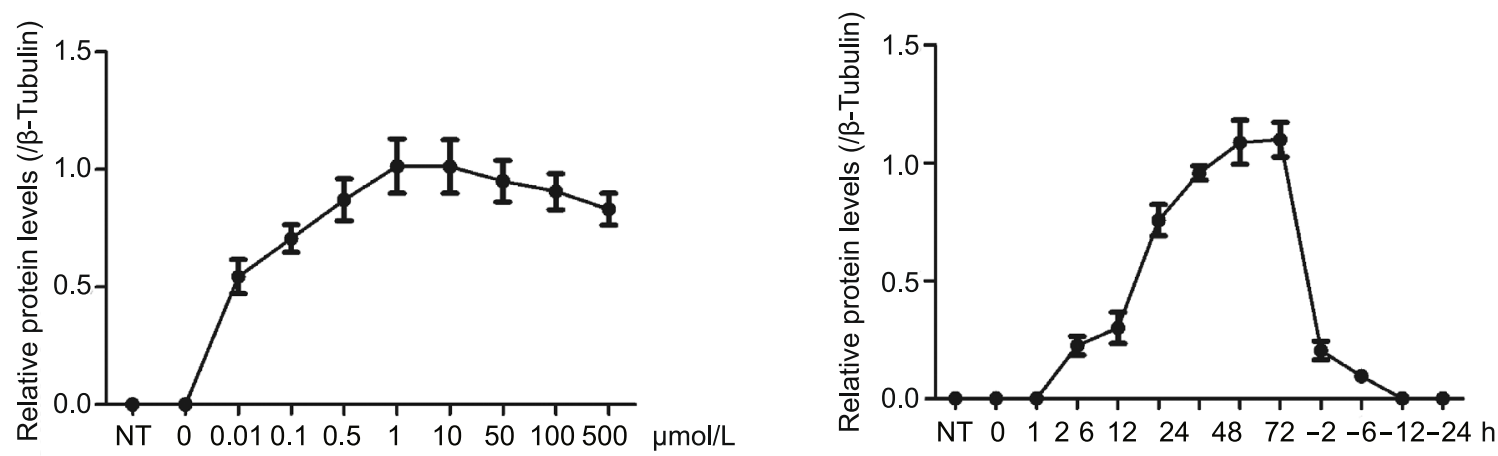
Figure 1. TMP-inducible DD-Cas9 constructs. (A) Schematic of the DD-Cas9 strategy. DD-Cas9 is inactive (degraded) in the absence of a cell-permeable small molecule trimethoprim (TMP), and is active (fully functional) in the presence of TMP. (B) DD-Cas9 variants. Top row, the constitutive wild-type Cas9 expression plasmid S9, which contains a CBh promoter, a Flag tag, two NLSs joined to Cas9 enzyme. Other rows represent five DD-Cas9 variants with distinct configurations of Flag tag, $\mathrm{DD}$, and Cas9 with their abbreviations on the left. PB3 and PB5, terminals of the piggyBac transposon; $\mathrm{pA}$, polyadenylation signal. (C) Representative T7EI cleavage assay of DD-Cas9 variants at TET1 locus. In HeLa cells, the editing activity of these DD-Cas9 variants was evaluated with and without $1 \mu \mathrm{mol} / \mathrm{L}$ TMP treatment for 5 days. Red asterisks indicate the expected T7El-specific cleavage bands. Relative indel frequencies (\%) were illustrated at the bottom line. L, $1 \mathrm{~kb}$ plus DNA ladder; NT, non-transfected group. (D) Bar charts illustrated the targeting efficiencies of TET1, TET2, TET3 by T7E1 assay in the absence or presence of TMP. F5D was tightly controlled by TMP and displayed significantly higher activity across all three targeted genes. Data indicate the means \pm SD of three biological replicates $\left({ }^{\star} P<0.05,{ }^{* \star} P<0.01,{ }^{* \star *} P<0.001\right.$, Student's $t$-test). (E) Determination of the optimal TMP working concentration of F5D. HeLa cells expressing F5D were exposed to TMP with increasing concentrations for 5 days. The Cas 9 protein levels were analyzed by Western blot with an anti-Flag antibody (top). $\beta$-Tubulin was used as a loading control. The corresponding protein levels were quantified using the ImageJ software, and the indicated ratios were graphed (bottom). The results show that Cas9 protein level reaches a plateau at $1 \mu \mathrm{mol} / \mathrm{L}$ TMP. Data indicate the means \pm SEM of three independent experiments. $(F)$ Kinetics of DD mediated Cas9 protein degradation. Western blot analysis was performed for DD-Cas9 proteins obtained from continuous TMP $(1 \mu \mathrm{mol} / \mathrm{L})$ exposure (up to $72 \mathrm{~h}$ ) or withdrawal after $2 \mathrm{~h}$ TMP treatment at indicated time points (top). The indicated ratios were graphed (bottom). Data indicate the means \pm SEM of three independent experiments..

DD to different domains of Cas 9 to form five variants with the purpose of tuning Cas9 expression at the protein level. Although F5D met our demands well both in vitro and in vivo, more configurations could be tested for other purposes. In conclusion, our successful in vivo delivery of an inducible CRISPR-Cas9 may provide a foundation for future safer gene therapy.

\section{FOOTNOTES}

We thank Dr. Xuguang Du, Dr. Tao Feng and Dr. Chunlong Xu for reading the manuscript. This work was supported by Transgenic Research Grant 2016ZX08010001, Transgenic Research Grant 2016ZX08009003, Beijing Advanced Innovation Center for Food Nutrition and Human Health, College of Biological Sciences, China Agricultural University, the Project for Extramural Scientists of State Key Laboratory of Agrobiotechnology Grant 2017SKLAB7-6, and the 111 Project (B12008).

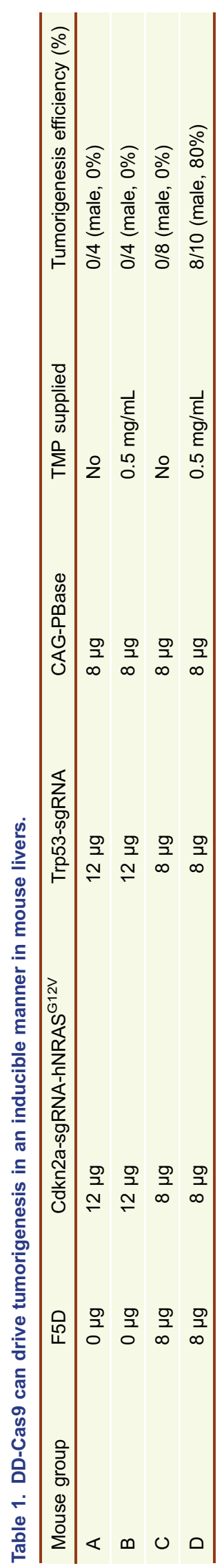


A

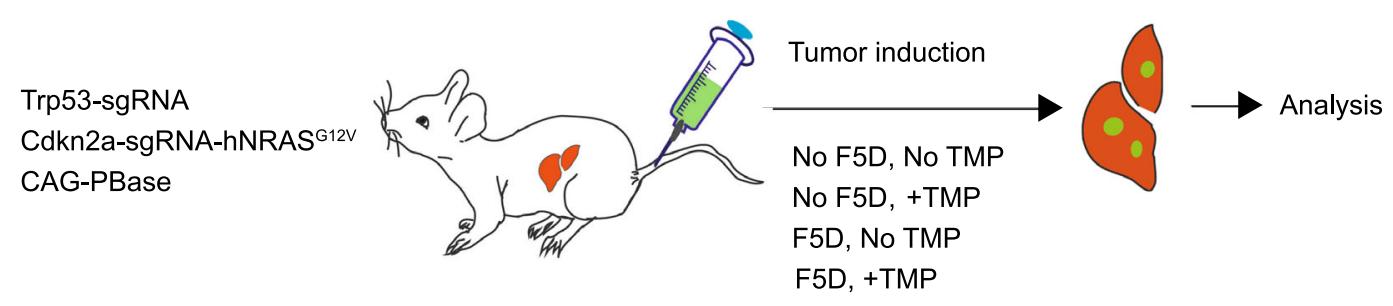

B

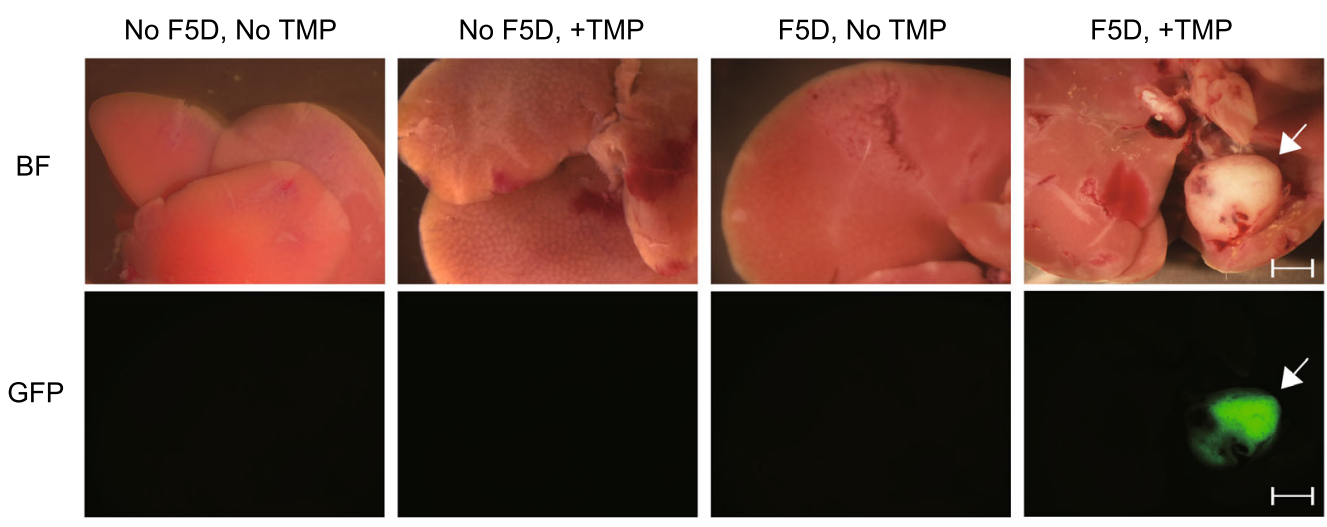

C

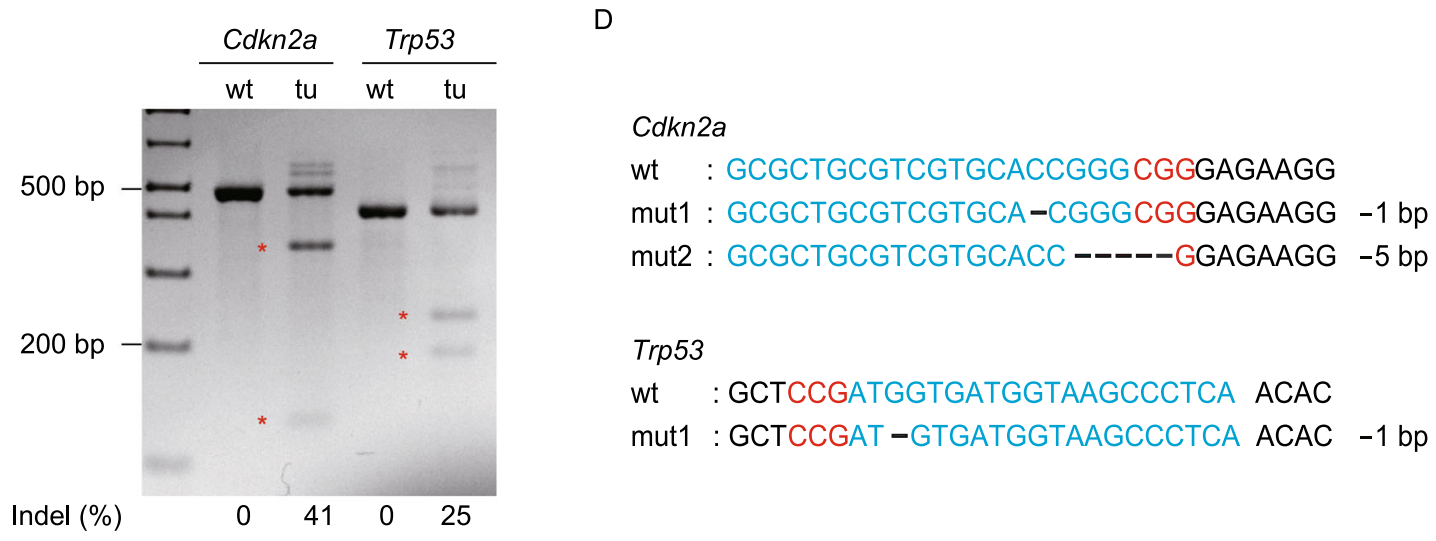

E

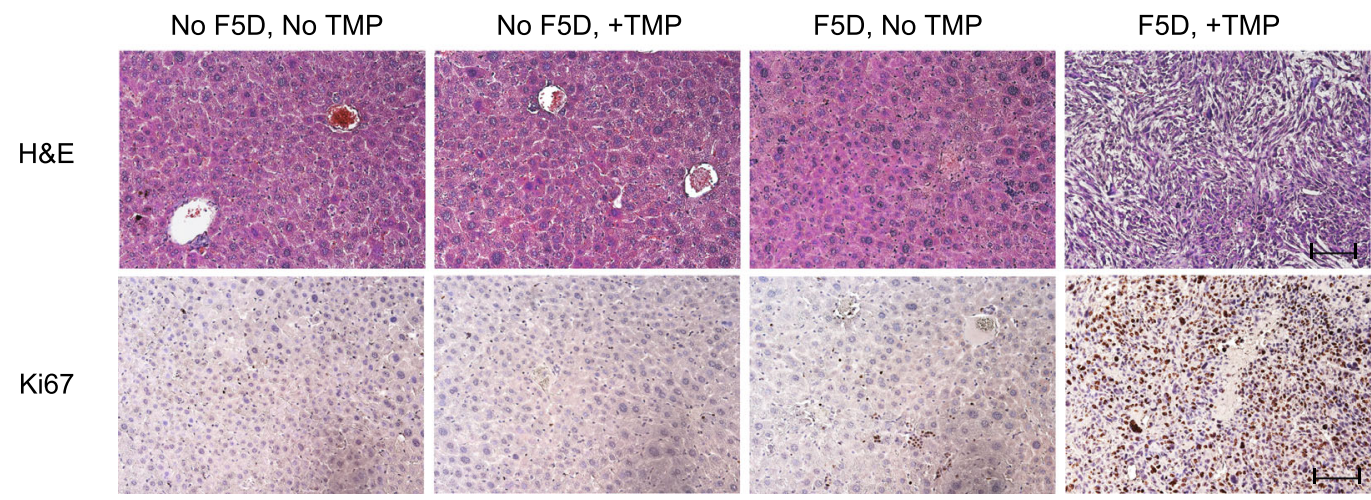


Figure 2. DD-Cas9 mediated liver tumorigenesis in an inducible manner. (A) Workflow of the induction of liver tumors in mice by hydrodynamic tail vein injection. (B) Representative liver tumors obtained from the mice under different treatments. Mice injected F5D and induced with TMP developed tumors with GFP fluorescence. White arrows indicate tumor sites. Scale bar, $100 \mu \mathrm{m}$. (C) T7EI cleavage assay of genome modification in a representative liver tumor. Red asterisks indicate the expected T7EI-specific cleavage bands used to quantify indel frequencies (the bottom line). Tu, tumor sample. (D) Sequences of indel mutation in a representative liver tumor. Protospacer-adjacent motif (PAM) sequences were labeled in red and sgRNA coding sequences were labeled in cyan. (E) H\&E and immunohistochemistry analysis of the indicated liver samples. Slides showed that tumor cells grew in a tubular pattern and proliferated rapidly by highly expressing Ki67 in contrast to the other liver tissues. Scale bar, $100 \mu \mathrm{m}$..

Sen Wu, Xiaomeng An and Linlin Li conceived and designed the experiments. Xiaomeng An performed the experiments. Xiaomeng An, Linlin Li and Sen Wu wrote the paper. Xiaomeng An and Linlin Li contributed equally to this article.

Xiaomeng An, Linlin Li, and Sen Wu declare that they have no conflict of interest. All institutional and national guidelines for the care and use of laboratory animals were followed.

\footnotetext{
Xiaomeng $\mathrm{An}^{1}$, Linlin $\mathrm{Li}^{2}$, Sen $\mathrm{Wu}^{1 凶}$

1 State Key Laboratory of Agrobiotechnology, College of Biological Sciences, China Agricultural University, Beijing 100193, China

2 State Key Laboratory of Veterinary Etiological Biology, National Foot and Mouth Diseases Reference Laboratory, Key Laboratory of Animal Virology of Ministry of Agriculture, Lanzhou Veterinary Research Institute, Chinese Academy of Agricultural Sciences, Lanzhou 730000, China

$\bowtie$ Correspondence: swu@cau.edu.cn (S. Wu)
}

\section{OPEN ACCESS}

This article is distributed under the terms of the Creative Commons Attribution 4.0 International License (http://creativecommons.org/ licenses/by/4.0/), which permits unrestricted use, distribution, and reproduction in any medium, provided you give appropriate credit to the original author(s) and the source, provide a link to the Creative Commons license, and indicate if changes were made.

\section{REFERENCES}

Balboa D, Weltner J, Eurola S, Trokovic R, Wartiovaara K, Otonkoski $\mathrm{T}$ (2015) Conditionally stabilized dCas9 activator for controlling gene expression in human cell reprogramming and differentiation. Stem Cell Rep 5:448-459

Dow LE, Fisher J, O'Rourke KP, Muley A, Kastenhuber ER, Livshits G, Tschaharganeh DF, Socci ND, Lowe SW (2015) Inducible in vivo genome editing with CRISPR-Cas9. Nat Biotechnol 33:390-U398

Iwamoto M, Bjorklund T, Lundberg C, Kirik D, Wandless TJ (2010) A general chemical method to regulate protein stability in the mammalian central nervous system. Chem Biol 17:981-988

Kleinjan DA, Wardrope C, Nga Sou S, Rosser SJ (2017) Drugtunable multidimensional synthetic gene control using inducible degron-tagged dCas9 effectors. Nat Commun 8:1191

Konermann S, Brigham MD, Trevino AE, Hsu PD, Heidenreich M, Cong L, Platt RJ, Scott DA, Church GM, Zhang F (2013) Optical control of mammalian endogenous transcription and epigenetic states. Nature 500:472-476

Liu KI, Ramli MNB, Woo CWA, Wang YM, Zhao TY, Zhang XJ, Yim GRD, Chong BY, Gowher A, Chua MZH et al (2016) A chemicalinducible CRISPR-Cas9 system for rapid control of genome editing. Nat Chem Biol 12:980-987

Lu J, Zhao C, Zhao Y, Zhang J, Zhang Y, Chen L, Han Q, Ying Y, Peng S, Ai R et al (2018) Multimode drug inducible CRISPR/ Cas9 devices for transcriptional activation and genome editing. Nucleic Acids Res 46:e25

Maji B, Moore CL, Zetsche B, Volz SE, Zhang F, Shoulders MD, Choudhary A (2017) Multidimensional chemical control of CRISPR-Cas9. Nat Chem Biol 13:9-11

Mali P, Yang LH, Esvelt KM, Aach J, Guell M, DiCarlo JE, Norville JE, Church GM (2013) RNA-guided human genome engineering via Cas9. Science 339:823-826

Sando R, Baumgaertel K, Pieraut S, Torabi-Rander N, Wandless TJ, Mayford M, Maximov A (2013) Inducible control of gene expression with destabilized Cre. Nat Methods 10:1085-1088

Senturk S, Shirole NH, Nowak DG, Corbo V, Pal D, Vaughan A, Tuveson DA, Trotman LC, Kinney JB, Sordella R (2017) Rapid and tunable method to temporally control gene editing based on conditional Cas9 stabilization. Nat Commun 8:1-10

Xu CL, Qi XL, Du XG, Zou HY, Gao F, Feng T, Lu HX, Li SL, An XM, Zhang LJ et al (2017) piggyBac mediates efficient in vivo CRISPR library screening for tumorigenesis in mice. Proc Natl Acad Sci USA 114:722-727

Zetsche B, Volz SE, Zhang F (2015) A split-Cas9 architecture for inducible genome editing and transcription modulation. Nat Biotechnol 33:139-142

Xiaomeng An and Linlin Li are Co-first authors.

Electronic supplementary material The online version of this article (https://doi.org/10.1007/s13238-018-0579-7) contains supplementary material, which is available to authorized users. 